\title{
THE NEUTRAL HYDROGEN DISTRIBUTION IN SPIRAL AND IRREGULAR GALAXIES
}

\author{
R. D. DAVIES \\ University of Manchester, Nuffield Radio Astronomy Laboratories, Jodrell Bank, \\ Cheshire, Great Britain.
}

\begin{abstract}
An investigation of several nearby and irregular galaxies shows significant changes in the neutral hydrogen distribution with morphological type. M31, an Sb, has a central hole while M33, an Sc, has relatively a much smaller hole or no hole at all and NGC 6822, an irregular galaxy, is centrally concentrated. The HII regions in all these morphological types are found in the regions of highest neutral hydrogen density.
\end{abstract}

Studies of nearby spiral and irregular galaxies have been undertaken at Jodrell Bank with sufficient angular and velocity resolution to make a comparison between the neutral hydrogen distributions in the two types of galaxy. Observations have been made with the $250 \mathrm{ft}$ radiotelescope illuminated to give a halfpower beamwidth of approximately $18^{\prime} \times 14^{\prime}$. The frequency resolution in the various surveys was $8.6 \mathrm{~km} \mathrm{~s}^{-1}$ or less. The spiral galaxies investigated were M31 (Sb), M33 (Sc) and M101 (Sc) and the irregular (Type I) galaxies were NGC 6822, IC 10 and IC 1613. The Sc galaxies, NGC 2403 and IC 342, have also been observed but the reduction of the data is not yet complete. The main discussion which follows will be directed towards M31, M33 and NGC 6822 where the angular resolution is best relative to the size of the galaxy.

\section{M31}

The integrated neutral hydrogen distribution in M31 has a central region of low surface density extending to about $5 \mathrm{kpc}$ from the centre (Roberts, 1967; Davies and Gottesman, 1970). Outside this lie ridges of high surface density that can be traced out to $30 \mathrm{kpc}$ from the centre. These ridges which are not fully resolved in the present observations are probably the counterparts of the neutral hydrogen 'spiral' arms in the Milky Way. The brightest ridges are associated with the spiral arms in M31 designated S4, N4, S5 and N5. A considerable amount of neutral hydrogen is found to be associated with the arms S6, N6, S7 and N7 which are fainter optically. Even beyond the optically identifiable arms there is significant neutral hydrogen emission.

A particular study has been made of the distribution of HII regions catalogued by Baade and Arp (1964) in relation to the distribution of neutral hydrogen. Firstly, on the scale of spiral arms these are found to lie in the regions of greatest neutral hydrogen density. Then, on a smaller scale $(\sim 2 \mathrm{kpc})$, within spiral arms the individual contour maps at fixed frequencies show concentrations which in most cases are correlated in position with groups of HII regions. This correlation is illustrated in the accompanying film which shows the sequence of neutral hydrogen maps at adjacent frequencies; these are superimposed on a plot of the distribution of $\mathrm{HII}$ regions. 
The original maps of M31 and also M33 are published by Gottesman and de Jager (1970). These data can be used to throw some light on the problem of the rate of star formation as a function of gas (neutral hydrogen) density. I am taking the surface density of $\mathrm{H}$ II regions in any given area as an index of the present rate of star formation in that area. The surface density of $\mathrm{H}$ i regions was compared with the neutral hydrogen surface density in 126 adjacent areas in a strip 15' wide along the major axis of M31 and the results are summarized in Figure 1. If a thickness of 200 pc is assumed

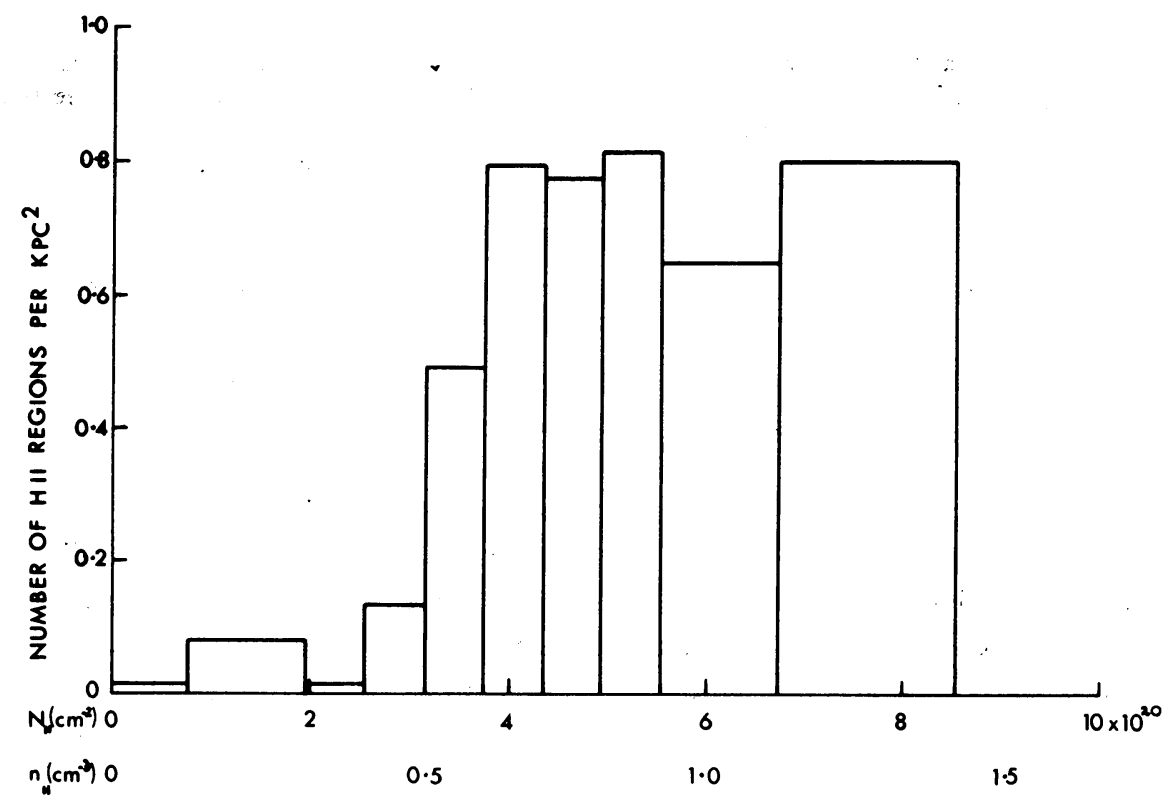

Fig. 1. The correlation between the surface density of $\mathrm{H}$ II regions and neutral hydrogen in M31. $N_{\mathrm{H}}$ is the surface density of neutral hydrogen projected onto the plane of M31 measured in atoms $\mathrm{cm}^{-2}$. $n_{\mathrm{H}}$ is the volume density calculated for an assumed thickness of $200 \mathrm{pc}$. Each block of the histogram represents an approximately equal area of M31.

for the neutral hydrogen layer the mean volume density $n_{\mathrm{H}}$, is as indicated on the abscissa. The main feature of Figure 1 is the apparent threshold in mean density at $0.5 \mathrm{~cm}^{-3}$ required before star formation begins. It was also found that the lack of Hir regions at low neutral hydrogen densities was characteristic of both the central regions and of the far outer regions. Remembering that these neutral hydrogen data were obtained with a beam which is $\sim 15^{\prime}$ in diameter, the threshold effect is remarkably clear; observations with higher angular resolution may well show an even more abrupt effect. Since the densities given here are the mean value as seen with this angular resolution we can expect that the local density at the Hil region where the stars are actually forming may be rather higher.

\section{M33}

The neutral hydrogen maps of M33 at different velocities have been added to give 
the surface density distribution projected onto the plane of the sky. This is shown in Figure 2 along with a schematic indication of the stellar and $\mathrm{H}$ il region distributions taken from the Sky Atlas prints and from Carranza et al. (1968). It is evident from Figure 2 that an angular resolution of $10^{\prime} \times 13^{\prime}$ (to which the original observations were deconvolved) is insufficient to resolve any central hole in the neutral hydrogen distribution of M33. Indeed the hole, if it exists, is smaller when scaled for the size of the galaxy than the hole in M31. Optically there is a distinct difference between

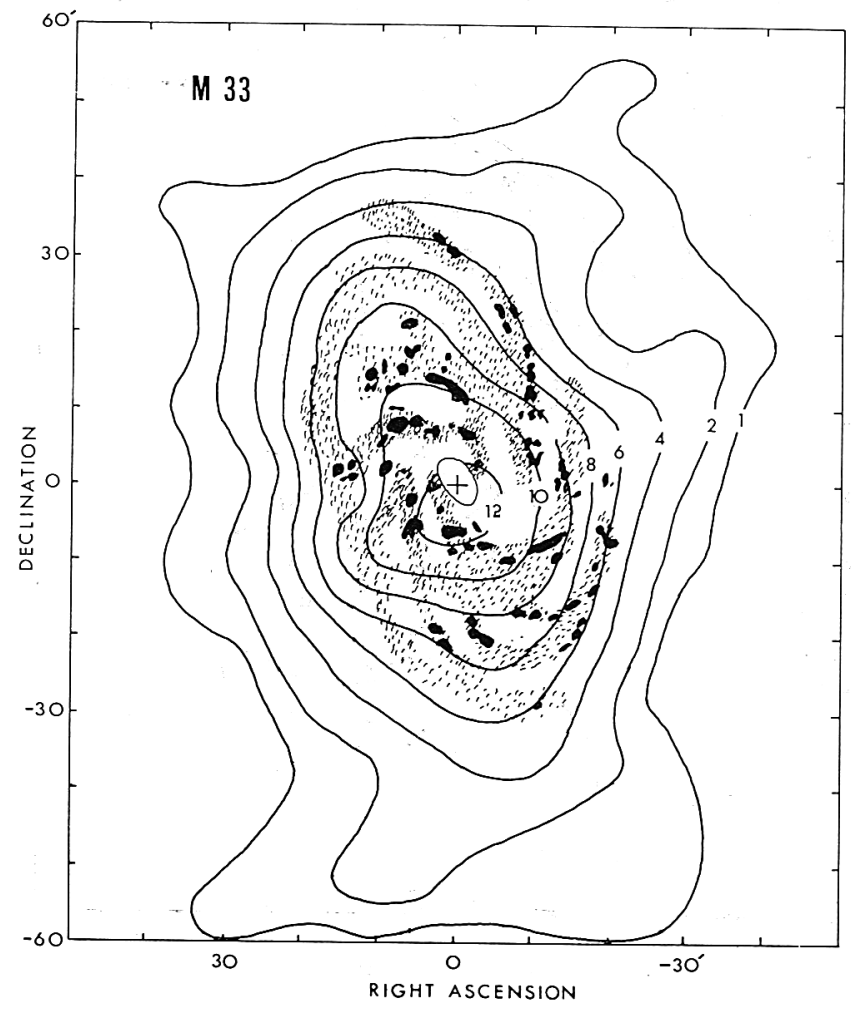

Fig. 2. The distribution of neutral hydrogen in M33 compared with the optical features. Hatched areas represent the principal stellar arms and the filled areas are the main $\mathrm{HII}$ regions. The contour levels are proportional to the surface density of neutral hydrogen.

the two galaxies in relation to the Popalation I distribution in the central regions. M33 has Hir regions scattered through the spiral arms and extending into the central regions in contrast to $\mathrm{M} 31$ which is devoid of Hil regions in its centre. The neutral hydrogen distribution in M33 appears therefore to follow the same general trend as the $\mathrm{H}$ II regions.

M33 has one unusual feature which will now be discussed. At velocities near the turnover velocity at either end of the rotation curve the neutral hydrogen is displaced in the clockwise sense from the major axis. This displacement can be seen even in 
the outer contour levels of the integrated neutral hydrogen distribution shown in Figure 2. By contrast the inner contours follow the optical shape very closely. The distortion in M33 is more clearly seen in the contour maps at fixed frequency. Two at either end of the spread in rotation velocity are illustrated in Figure 3 and show that the main concentration lies on the major axis but substantial wings of emission lie displaced clockwise from the main concentration in areas where there are no optical

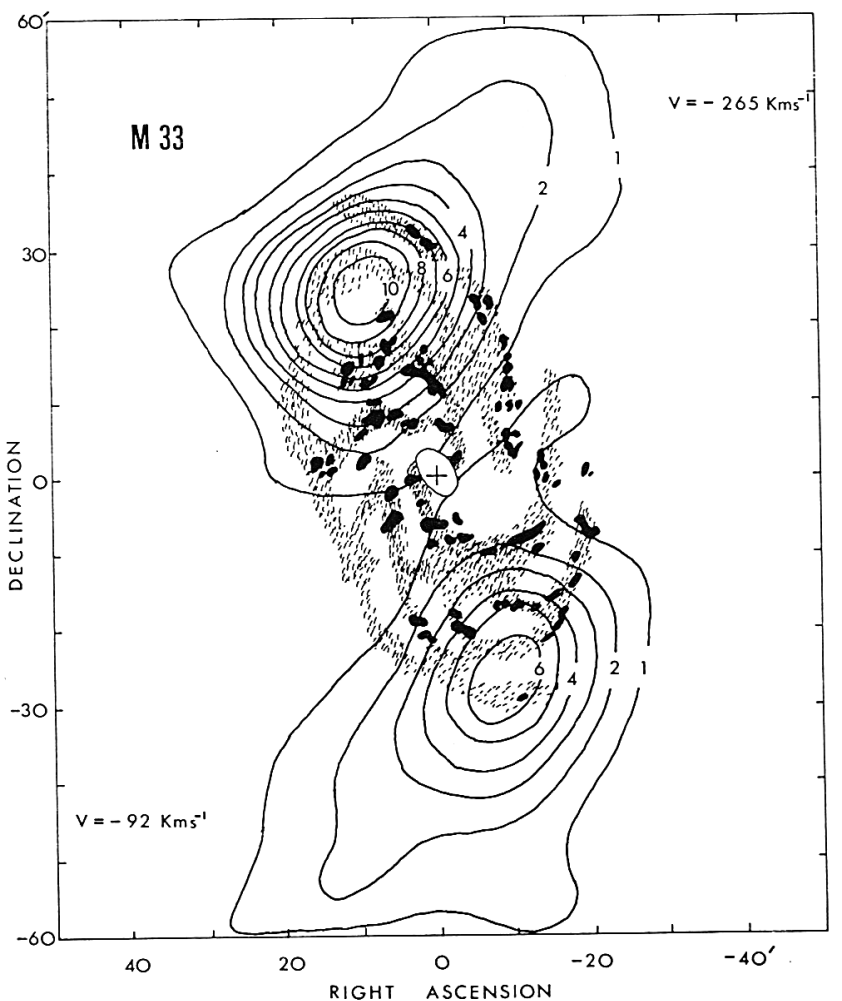

Fig. 3. Contour maps at fixed frequencies demonstrating the distortion in the neutral hydrogen distribution of M33. The northern contours are for a velocity of $-265 \mathrm{~km} \mathrm{~s}^{-1}$ and the southern contours are for $-92 \mathrm{~km} \mathrm{~s}^{-1}$. Each map plots the measured signal in a band $8.6 \mathrm{~km} \mathrm{~s}^{-1}$ wide.

features. At present the origin of these distortions in the neutral hydrogen is conjectural. They may represent an exaggerated form of the tilt in the plane seen in the Milky Way and proposed to explain certain features of the optical and neutral hydrogen structure of M31 (see, for example, Davies and Gottesman, 1970). This tilt is often believed to originate in gravitational tides amplified by a resonance effect in the companion galaxy. The most likely disrupting galaxy for M33 would seem to be M31 although a direct gravitational tide is insufficient to produce the observed effect. Further amplification is required as is the case for the Milky Way. 


\section{NGC 6822}

NGC 6822 is an Irr I galaxy with optical dimensions $20^{\prime} \times 20^{\prime}$ as given by Holmberg (1958). Its neutral hydrogen emission covers a much larger area as may be seen from Figure 4 and can be traced over an elliptical region at least $59^{\prime} \times 28^{\prime}$ (after removal of the beam smearing effect) with a major axis at position angle $118.5^{\circ} \pm 2.5^{\circ}$. The bright central optical region is elongated at an angle of about $70^{\circ}$ to the main neutral hydrogen concentration and is suggestive of a bar rather like the main body of the Large Magellanic Cloud. In fact the faint stellar extensions from the main bar of NGC 6822 could be embryo spiral arms condensing out of the main mass of neutral

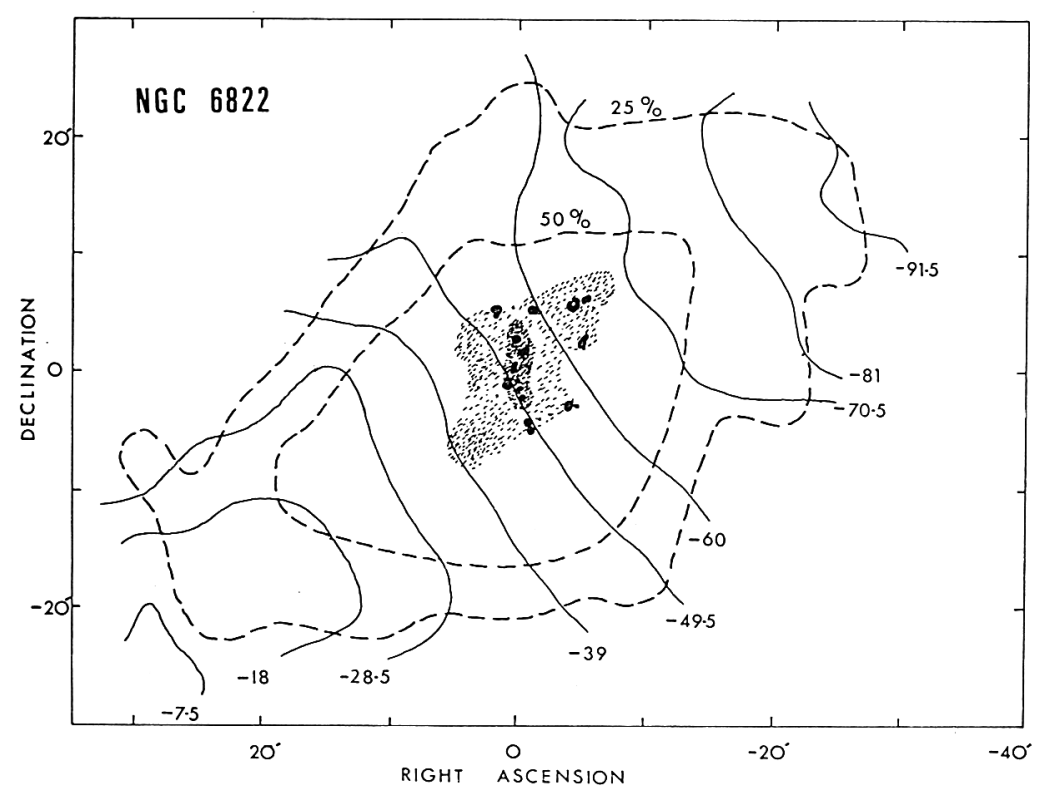

Fig. 4. The distribution of neutral hydrogen in NGC 6822 compared with optical features. The dashed contours represent neutral hydrogen surface density levels at 25 and $50 \%$ of the maximum while the full lines are lines of constant median velocity measured in $\mathrm{km} \mathrm{s}^{-1}$ relative to the Sun.

Hatched areas show the main distribution of stars and the filled areas are $\mathrm{H}$ II regions.

hydrogen. Several HII regions in NGC 6822 have been catalogued by Hodge (1969) and these, as shown in Figure 4, are confined to the main optical regions of the galaxy.

Evidently star formation is only proceeding at a significant rate in the central regions of the NGC 6822 protogalaxy. The surface density of neutral hydrogen in this central region of star formation is $\sim 2 \times 10^{21} \mathrm{~cm}^{-2}$ which, for an assumed line of sight thickness of $200 \mathrm{pc}$, corresponds to a density of $\sim 1.5 \mathrm{~cm}^{-3}$. If the true axial ratio of irregular galaxies is as found by Hodge and Hitchcock (1966) this density becomes $\sim 0.3 \mathrm{~cm}^{-3}$. At densities of half this value or less no star formation seems to have occurred. 


\section{Large Magellanic Cloud}

The Large Magellanic Cloud will be mentioned here because its neutral hydrogen structure is intermediate between that of M33 and NGC 6822. It is an irregular galaxy in which Hil regions and older stars are spread throughout the neutral hydrogen distribution (see, for example; McGee and Milton, 1966). Because of its closeness detailed comparisons can be made between the neutral hydrogen and stellar distributions which show the presence of Hil regions only in the densest knots of neutral hydrogen. This situation is similar to that found in M31, the only other galaxy studied with sufficiently high resolution to distinguish the concentrations of neutral hydrogen.

\section{Conclusion}

An investigation of the neutral hydrogen distribution relative to the $\mathrm{HII}$ and stellar distributions in M31, M33, the LMC and NGC 6822 shows a progression from an $\mathrm{HI}_{\mathrm{I}}$ and $\mathrm{HII}$ distribution with a central hole, through one with a more uniform distribution over the galaxy to one in which the $\mathrm{HI}$ and $\mathrm{H}_{\mathrm{II}}$ have a strong central distribution. Moreover the $\mathrm{HII}$ regions are found principally within the regions of higher neutral hydrogen density. It appears that averaged neutral hydrogen volume densities of 0.5 to $2 \mathrm{~cm}^{-3}$ are required for star formation.

\section{References}

Baade, W. and Arp, H.: 1964, Astrophys. J. 139, 1027.

Carranza, G., Courtès, G., Georgelin, Y., Monnet, G., and Pourcelot, A.: 1968, Ann. Astrophys. 31, 63.

Davies, R. D. and Gottesman, S. T.: 1970, Monthly Notices Roy. Astron. Soc. 149, 237.

Gottesman, S. T., and Davies, R. D.: 1970, Monthly Notices Roy. Astron. Soc. 149, 263.

Gottesman, S. T. and de Jager, G.: 1970, Mem. Roy. Astron. Soc. 74, 67.

Hodge, P. W. and Hitchcock, J. L.: 1966, Publ. Astron. Soc. Pacific 78, 79.

Hodge, P. W.: 1969, Astrophys. J. Suppl. 18, 73.

Holmberg, E.: 1958, Medd. Lunds Astron. Obs., Ser. II, No. 136.

McGee, R. X. and Milton, J. A.: 1966, Australian J. Phys. 19, 343.

Roberts, M. S.: 1967, in H. van Woerden (ed.), 'Radio Astronomy and the Galactic System', $I A U$ Symp. 31, 189.

\section{Discussion}

Mrs Rubin: From your observations, have you determined a rotation curve for M31? Does it agree with the optical rotation curve? Is there velocity asymmetry between the NE and SW sides of the galaxy?

Davies: Yes, we have a rotation curve for M31 which agrees within the accuracy of both sets of data with the optical data from various sources. There is a small and apparently significant difference between the northern and southern major axes. We do not find asymmetry of more than $20 \mathrm{~km} \mathrm{~s}^{-1}$. between the preceding and following sides of the major axes. These results are discussed by Gottesman and myself in the reference given in my paper.

Arp: Are the occasional protuberances in the hydrogen profiles: in M31, particularly along the minor axis, real? If you consider them real, what interpretation do you give to them?

Davies: These effects show only in the outer contour in each fixed velocity contour map which is only twice the rms noise level. Consequently, only small weight should be assigned to them in the 
M31 maps. The distortions discussed in the text for M33 are certainly real; I have already suggested an interpretation for them.

Rees: The apparent sharp cut-off in the star formation rate when $n_{\mathrm{H}}$ falls below $\sim 0.5 \mathrm{~cm}^{-3}$ may perhaps be taken as evidence for the 'two phase' model of the interstellar medium discussed by, for example, Field et al. In this picture, when the density is below a critical density (whose value depends on the heating rate) all the matter is in the hot phase and no cool clouds can exist.

de Vaucouleurs: We have radial velocities for about $15 \mathrm{HII}$ region in M101 from spectrum scans of $\mathrm{N}_{1}, \mathrm{~N}_{2}$, at McDonald Observatory which may help in the analysis of the $21-\mathrm{cm}$ data.

What is the position angle of the line of nodes from the $21-\mathrm{cm}$ velocities?

Rogstad: $30^{\circ}$ to $40^{\circ}-45^{\circ}$, depending on the interpretation of the $21-\mathrm{cm}$ velocity map.

Weliachew: I would like to say a few words about a paper by M. Guélin and myself that is to be published in the next issue of Astron. Astrophys., about the neutral hydrogen in M101. The main features which have come out of this study are (1) the asymmetry in hydrogen content between northern and southern halves as already noted by other observers, and (2) large-scale agreement between projected hydrogen density maxima and main spiral features. 